\title{
Montanide, Poly I:C and nanoparticle based vaccines promote differential suppressor and effector cell expansion: a study of induction of CD8 T cells to a minimal Plasmodium berghei epitope
}

\author{
Kirsty L. Wilson, Sue D. Xiang* and Magdalena Plebanski* \\ Department of Immunology, Central Clinical School, Faculty of Medicine, Nursing and Health Sciences, Monash University, Melbourne, VIC, Australia
}

\section{Edited by:}

Urszula Krzych, Walter Reed Army

Institute of Research, USA

\section{Reviewed by:}

Vasso Apostolopoulos, Victoria University, Australia

Stasya Zarling, Walter Reed Army Institute of Researach, USA

\section{*Correspondence:}

Magdalena Plebanski and Sue D. Xiang, Department of Immunology, Central Clinical School, Faculty of Medicine, Nursing and Health Sciences, Monash University, 89 Commercial Road, Melbourne, VIC 3004, Australia

e-mail:magdalena.plebanski@ monash.edu

sue.xiang@monash.edu
The development of practical and flexible vaccines to target liver stage malaria parasites would benefit from an ability to induce high levels of CD8 T cells to minimal peptide epitopes. Herein we compare different adjuvant and carrier systems in a murine model for induction of interferon gamma (IFN- $\gamma$ ) producing CD8 T cells to the minimal immunodominant peptide epitope from the circumsporozoite protein (CSP) of Plasmodium berghei, pb9 (SYIPSAEKI, referred to as KI). Two pro-inflammatory adjuvants, Montanide and Poly I:C, and a non-classical, non-inflammatory nanoparticle based carrier (polystyrene nanoparticles, PSNPs), were compared side-by-side for their ability to induce potentially protective CD8T cell responses after two immunizations. KI in Montanide (Montanide + KI) or covalently conjugated to PSNPs (PSNPs-KI) induced such high responses, whereas adjuvanting with Poly I:C or PSNPs without conjugation was ineffective. This result was consistent with an observed induction of an immunosuppressed environment by Poly I:C in the draining lymph node (dLN) 48 h post injection, which was reflected by increased frequencies of myeloid derived suppressor cells (MDSCs) and a proportion of inflammation reactive regulatory T cells (Treg) expressing the tumor necrosis factor receptor 2 (TNFR2), as well as decreased dendritic cell (DC) maturation. The other inflammatory adjuvant, Montanide, also promoted proportional increases in the TNFR2 ${ }^{+}$Treg subpopulation, but not MDSCs, in the dLN. By contrast, injection with non-inflammatory PSNPs did not cause these changes. Induction of high CD8 T cell responses, using minimal peptide epitopes, can be achieved by non-inflammatory carrier nanoparticles, which in contrast to some conventional inflammatory adjuvants, do not expand either MDSCs or inflammation reactive Tregs at the site of priming.

Keywords: malaria, adjuvant, nanoparticle, CD8 peptide, Treg, MDSC

\section{INTRODUCTION}

Malaria affects over 200 million people annually, resulting in over half a million deaths with most mortality coming from infections with Plasmodium falciparum, and developing a malaria vaccine has become a major global effort (Arama and Troye-Blomberg, 2014). The most advanced malaria vaccine development focuses on the pre-erythrocytic stage, at which sporozoite parasites enter the circulation after a mosquito bite and then rapidly enter and infect hepatocytes. CD8 T lymphocytes, particularly those capable of producing interferon gamma (IFN- $\gamma$ ), can mediate effective sterile liver-stage immunity (Schneider et al., 1999; Doolan and Martinez-Alier, 2006; Krzych et al., 2014). Developing a CD8 T cell inducing liver-stage vaccine would be beneficial to further avoid the clinical symptoms of malaria, such as fever, associated with subsequent blood stages of infection, as well as preventing transmission and the sexual development of parasites (Arama and Troye-Blomberg, 2014). Whole irradiated sporozoites are effective CD8 T cell inducing vaccines (Doolan and Martinez-Alier, 2006), and immunity to a dominant circumsporozoite protein
(CSP) CD8 T cell epitope of $P$. berghei, named pb9 (sequence SYIPSAEKI), can mediate protection in murine animal models (Schneider et al., 1999).

Unfortunately, synthetic and recombinant vaccines have been less effective at inducing CD8 T cells, particularly in humans (Arama and Troye-Blomberg, 2014). The choice of adjuvant and the delivery system for the selected antigens will play a major role in the ability of vaccines to induce CD8 T cell immunity. Minimal CD8 $\mathrm{T}$ cell peptide epitopes offer production, stability, and flexibility advantages in vaccine formulation (Plebanski et al., 2006). Herein we compare side by side two adjuvants with proven capacity to promote CD8 T cell responses, Montanide (a water in oil emulsion) and Poly I:C (TLR3 agonist). Both have been used in various clinical trials as adjuvants in human vaccines against specific diseases (Aucouturier et al., 2002; Bonhoure and Gaucheron, 2006; Trumpfheller et al., 2008; Longhi et al., 2009; Mbow et al., 2010). Given cerebral malaria pathology is associated with inflammation (Postels and Birbeck, 2013), the use of novel nanovaccine technologies which induce CD8 T cell immunity 
without conventional pro-inflammatory signals also offers a conceptual advantage. Based on our previous studies, such inert nanoparticles coated with a target antigen of choice can promote high levels of immunity in the absence of inflammation or added extrinsic adjuvants, even to peptide based antigens (Fifis et al., 2004a,b; Xiang et al., 2013). Responses are as high as experimental gold standards for antibody production (e.g., Freunds adjuvant) and CD8 T cell induction [e.g., ex-vivo antigen pulsed dendritic cells (DCs) ], and better than a range of conventional inflammatory experimental adjuvants (Fifis et al., 2004a).

The size of the nanoparticle is a key factor, with even small deviations away from the optimal size range of $40-50 \mathrm{~nm}$ causing major decreases in immunogenicity (Fifis et al., 2004a; Mottram et al., 2007). We herein compared Montanide and Poly I:C, representing two pro-inflammatory adjuvants, against such nanoparticle based vaccines for delivery of the minimal pb9 CD8 $\mathrm{T}$ cell epitope. Moreover, we speculated that inflammatory responses during the priming phase of immunity could further result in the activation of the immune-suppressive mechanisms that arise to control such inflammation, but may interfere with efficient CD8 T cell stimulation. In this context, it is known that enhancing cross-presenting DC frequency and function, and preventing myeloid derived suppressor cells (MDSCs) accumulation promotes antigen specific immune responses (Ohkusu-Tsukada et al., 2011). It has also been suggested that Poly I:C is capable of increasing antigen specific effector $\mathrm{T}$ cells over regulatory $\mathrm{T}$ cells (Treg), enhancing immunity (Perret et al., 2013). Hence, as well as comparing the magnitude of the CD8 T cell responses induced by the different adjuvants, this study evaluates the ability of Montanide, Poly I:C, and nanoparticles to promote the induction of inflammation reactive Tregs and the expansion of MDSCs, compared to effector T cells and stimulatory antigen presenting types such as DCs.

\section{MATERIALS AND METHODS MICE}

Six to eight weeks old BALB/c mice were purchased from Monash Animal Services (MAS) Melbourne, VIC, Australia. The studies presented here were approved by the Alfred Medical Research and Education Precinct (AMREP) Animal Ethics Committee, Melbourne, VIC, Australia.

\section{NANOVACCINE FORMULATIONS}

Conjugation of malaria peptide antigens to nanoparticles was based on the previous described method (Xiang et al., 2013) with a slight modification. Briefly, carboxylated polystyrene nanoparticles (PSNPs; Polysciences Inc, Warrington, PA, USA) of $40 \mathrm{~nm}$ $(\sim 40-50 \mathrm{~nm})$ at a final of $1 \%$ solids were activated in a mixture containing 2-N-Morpholino-ethanesulfonic acid (MES; $50 \mathrm{mM}$ final, $\mathrm{pH}=7$ ), and 1-ethyl-3-(3-dimethylaminopropryl) carbodiimide hydrochloride (EDC; $4 \mathrm{mg} / \mathrm{ml}$ final). Malaria peptide SYIPSAEKI (KI; Mimotopes, Melbourne, VIC, Australia; $1 \mathrm{mg} / \mathrm{ml}$ final) was also added to the conjugation mix and together incubated on a rotary wheel at room temperature for approximately $4 \mathrm{~h}$. Following antigen incubation, the conjugation reactions were then quenched by adding excess glycine $(7 \mathrm{mg} / \mathrm{ml}$ final $)$ and further incubated for $30 \mathrm{~min}$. The free, unconjugated peptide antigens, and other excess conjugation agents, were removed by dialysis (10-14 kDa molecular weight cut-off (MWCO) membrane; Viskase, Darien, IL, USA) against phosphate buffered solution ( $\mathrm{PBS}, \mathrm{pH}=7.2$ ) at $4^{\circ} \mathrm{C}$ overnight. Conjugation efficiency and final sizes of the nanovaccine formulation (e.g., PSNPsKI) were determined by Bicinchoninic acid assay (BCA; Thermo Fisher Scientific, Rockford, IL, USA) and dynamic light scattering instruments (Zetasizer; Malvern Instruments, Worcestershire, UK), respectively, following the manufacture's instruction.

\section{OTHER VACCINE ADJUVANT AND IMMUNIZATIONS}

Other vaccine adjuvants such as Montanide ISA $720(70 \% \mathrm{v} / \mathrm{v}$ final, Tall Bennett Group, USA), Polyinosinic-polycytidylic acid sodium salt (Poly I:C; $25 \mu \mathrm{g} /$ mouse final, Sigma Aldrich, St. Louis, MO, USA) were also used in this study. The adjuvant effect of these vaccine formulations were tested in vivo by immunization of mice and measuring for IFN- $\gamma$ production by ELISpot assay (Xiang et al., 2006). Briefly, adjuvant mixed KI formulations (e.g., $\mathrm{KI}+$ Montanide; KI + PolyI:C; KI + PSNPs) at the desired final concentrations ( $\sim 25 \mu \mathrm{g} \mathrm{KI} /$ mouse) and nanoparticle conjugated KI (PSNPs-KI at $1 \%$ solid of PSNPs) formulations were injected into mice intradermally (i.d.) at the base of the tail. 14 days after the last immunization, mice were sacrificed and splenocytes were isolated and assayed for IFN $-\gamma$ production via ELISpot assay.

\section{ELISPOT ASSAY}

Antigen specific CD8 $\mathrm{T}$ cell responses were evaluated by IFN- $\gamma$ ELISpot assay (Xiang et al., 2006). Briefly, 96 well multiscreen filter plates (MSIP plates, Millipore, Billerica, MA, USA) were coated with $5 \mu \mathrm{g} / \mathrm{ml}$ final $(100 \mu \mathrm{l} /$ well) of anti-mouse IFN- $\gamma$ (AN18, MABTech, Stockholm, Sweden) in PBS, and incubated overnight at $4^{\circ} \mathrm{C}$. Following overnight incubation, the wells were washed and blocked with RPMI 1640 (Gibco, Life Technologies, Carlsbad, CA, USA), supplemented with $10 \%$ fetal calf serum (FCS; Gibco, Life Technologies), 100 units $/ \mathrm{ml}$ penicillin, $100 \mu \mathrm{g} / \mathrm{ml}$ streptomycin, $2 \mathrm{mM} \mathrm{L-glutamine,} 1 \mathrm{M}$ Hepes, and $0.1 \mathrm{mM} \mathrm{2-}$ mercaptoethanol, for a minimum of $1 \mathrm{~h}$. Splenocytes from mice (immunized with or without vaccine formulations as listed above) were added in triplicate wells $\left(1 \times 10^{7}\right.$ cells $\left./ \mathrm{ml}, 50 \mu \mathrm{l} / \mathrm{well}\right)$, along with the recall antigen (peptide SYIPSAEKI at different doses, $50 \mu \mathrm{l} /$ well). Media alone control, or concanavalin A (ConA; Amersham Biosciences, Uppsala, Sweden; final $1 \mu \mathrm{g} / \mathrm{ml}$ ) were also used, as negative or positive controls, respectively. All mice produced high levels of IFN- $\gamma$ in response to ConA, with SFU often above the threshold for accurate counting (data not shown), indicating adequate cell viability and functionality. Cells with antigens were incubated in a $37^{\circ} \mathrm{C}$ incubator filled with $6 \% \mathrm{CO}_{2}$ for a minimum of $16 \mathrm{~h}$. Plates were then washed five times in PBS, biotinylated detection antibody anti-IFN- $\gamma$ (R4-6A2-Biotin, MABTech; $1 \mu \mathrm{g} / \mathrm{ml}$ in PBS 0.5\% FCS, $100 \mu \mathrm{l} /$ well) was added and followed by further incubation at room temperature for $2 \mathrm{~h}$. Plates were then washed again, as above, and streptavidinalkaline phosphatase enzyme conjugate (ALP; $1 \mu \mathrm{g} / \mathrm{ml}$ in PBS $0.5 \%$ FCS, $100 \mu \mathrm{l} /$ well; MABTech) was added, followed by a further $1.5 \mathrm{~h}$ incubation at room temperature. After a final wash in PBS and followed by water, the spots were developed using a colorimetric AP kit (Bio-Rad, Philadelphia, PA, USA) following the manufacturer's instructions. Spots were counted by an 
AID ELISpot Reader System (Autoimmun Diagnostika GmbH, Germany).

\section{FLOW CYTOMETRY}

For phenotypic analysis of cells by flow cytometry, inguinal lymph node cells were isolated $48 \mathrm{~h}$ after immunization with the adjuvants alone. $2 \times 10^{6}$ cells/sample were stained for $15 \mathrm{~min}$ at room temperature with $30 \mu \mathrm{l}$ of antibody cocktails, including antibodies with different fluorochromes at different concentrations based on prior optimizations. Antibodies used in the present study include; anti-CD11c V450 (HL3), anti-CD11b PeCy7 (M1/70), anti-Gr1 (Ly6C and Ly6G) PerCP Cy5.5 (RB6-8C5), anti-CD3 AF700 (500A2), anti-CD4 BV605 (RM4-5, Biolegend, San Diego, CA, USA), anti-CD8 BV650 (53-6.7, Biolegend), anti-CD25 PeCy7 (PC61), anti-FoxP3 APC (MF23), and anti-CD120b (TNFR2) PE (TR75-89). All antibodies were from BD Biosciences (NJ, USA) except where specifically indicated. Following incubation, cells were washed with $100 \mu \mathrm{l}$ PBS/2\% FCS (FACS buffer). Stained cells were fixed with $1 \%(\mathrm{v} / \mathrm{v})$ paraformaldehyde (PFA, Sigma Aldrich) and acquired using an LSRII flow cytometer (BD Biosciences) located at the AMREP Flow Cytometry Core Facility (Melbourne, VIC, Australia). Data was analyzed using FlowJo software (version10, Treestar, USA).

\section{STATISTICAL ANALYSIS}

Statistical analysis was done by ANOVA analysis, with post hoc Tukeys multiple comparison tests or Fisher's LSD test, or unpaired $t$-tests, using Graphpad Prism software (version 6, San Diego, CA, USA). Statistical significance was determined as $p<0.05$. Group sizes are indicated in the figure legends. All values are expressed as mean \pm SD.

\section{RESULTS \\ PEPTIDE COVALENTLY BOUND TO, BUT NOT MIXED WITH, PSNPS INDUCES CD8 T CELLS}

Peptide delivery by nanoparticles (either mixed or conjugated) was compared for immunogenicity in vivo using BALB/c mice. To generate the conjugated nanovaccine, the immune-dominant CD8 T cell peptide epitope of the CSP protein, SYIPSAEKI (KI), from $P$. berghei was covalently attached to carboxylated polystyrene nanoparticles (PSNPs, 40-50 nm) using an optimized covalent conjugation protocol as previously described (Xiang et al., 2013). As shown in Table 1, the average size of the PSNPs-KI formulation was $47.97 \pm 2.64 \mathrm{~nm}$, and the polydispersity index (PdI) was very low $(0.07 \pm 0.03)$, indicating the successful formulation of a uniformly dispersed nanoparticle formulation with a narrow size distribution range (Figure 1). The antigen loading

Table 1 | Characterization of SYIPSAEKI conjugation to PSNPs (PSNPs-KI) for size, polydispersity, and peptide loading*.

\begin{tabular}{llll}
\hline Formulation & Size $(\mathbf{n m})$ & $\begin{array}{l}\text { Polydispersity } \\
\text { index }(\text { Pdl) }\end{array}$ & $\begin{array}{l}\text { Peptide molecules } \\
\text { per particle }\end{array}$ \\
\hline PSNPS-KI & $47.97 \pm 2.64$ & $0.07 \pm 0.03$ & $1032.6 \pm 147.8$
\end{tabular}

${ }^{*}$ Data presented as mean $\pm S D, n=4$ repeated conjugations.

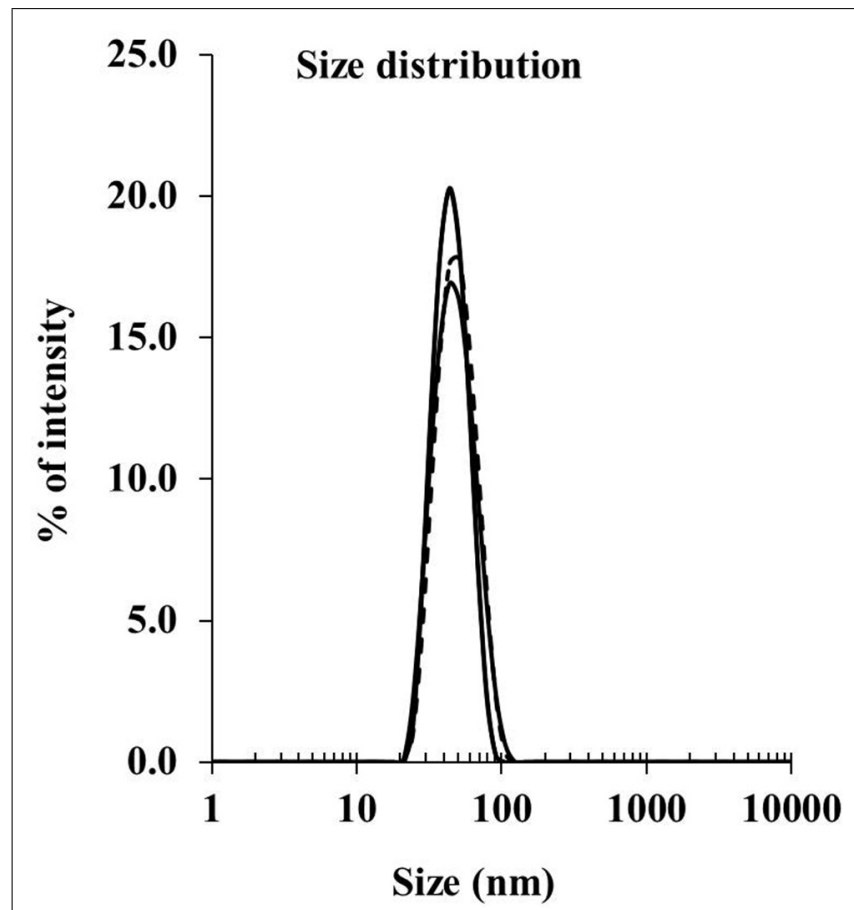

FIGURE 1 | Size distribution for PSNPs-KI formulations. SYIPSAEKI peptides were covalently conjugated to PSNPs, and the final sizes were measured by dynamic light scattering instruments (Zetasizer).

was $0.32 \pm 0.09 \mathrm{mg} / \mathrm{ml}$, which represented $1032.6 \pm 147.8$ peptide molecules per particle (Table 1 ). The number of peptide molecules per particle was comparable to previous studies with a model peptide antigen, SIINFEKL, where potent responses were observed at that loading (Xiang et al., 2013).

Mice were immunized twice, 14 days apart, with SYIPSAEKI peptide either alone (KI alone), mixed with the PSNPs (PSNPs $+\mathrm{KI}$ ), or covalently conjugated to the PSNPs (PSNPs-KI) at the dosage of $\sim 25 \mu \mathrm{g}$ of $\mathrm{KI} / \mathrm{mouse} /$ injection. As results show in Figure 2A, neither the "KI alone" nor the "PSNPs + KI" treatment groups showed induction of KI specific CD8 T cell responses, assessed by IFN- $\gamma$ ELISpot after two immunizations. However, when mice were immunized with KI conjugated to PSNPs (PSNPs$\mathrm{KI})$, significant $(p<0.001)$ levels of KI specific IFN- $\gamma$ producing CD8 $\mathrm{T}$ cells were induced, even at the smallest amount of recall antigen concentration $(0.25 \mu \mathrm{g} / \mathrm{ml})$. Increasing the recall antigen concentration did not further enhance the overall antigen specific IFN- $\gamma$ responses, suggesting the recall of high affinity $T$ cells. These results also show that this specific malaria antigen peptide needs to be covalently conjugated to its carrier nanoparticles to induce potent immune responses. Moreover, it shows that this system can utilize minimal CD8 T cell epitopes, without added CD4 T cell epitopes, and still induce levels of immune responses previously associated with powerful 'Prime-boost' immunization modalities and sterile protection against sporozoite challenge (Plebanski et al., 1998).

Given the PSNPs-KI formulation induced potent immune responses with two immunizations; we further tested formulation potency in a single dose immunization regime. As shown 

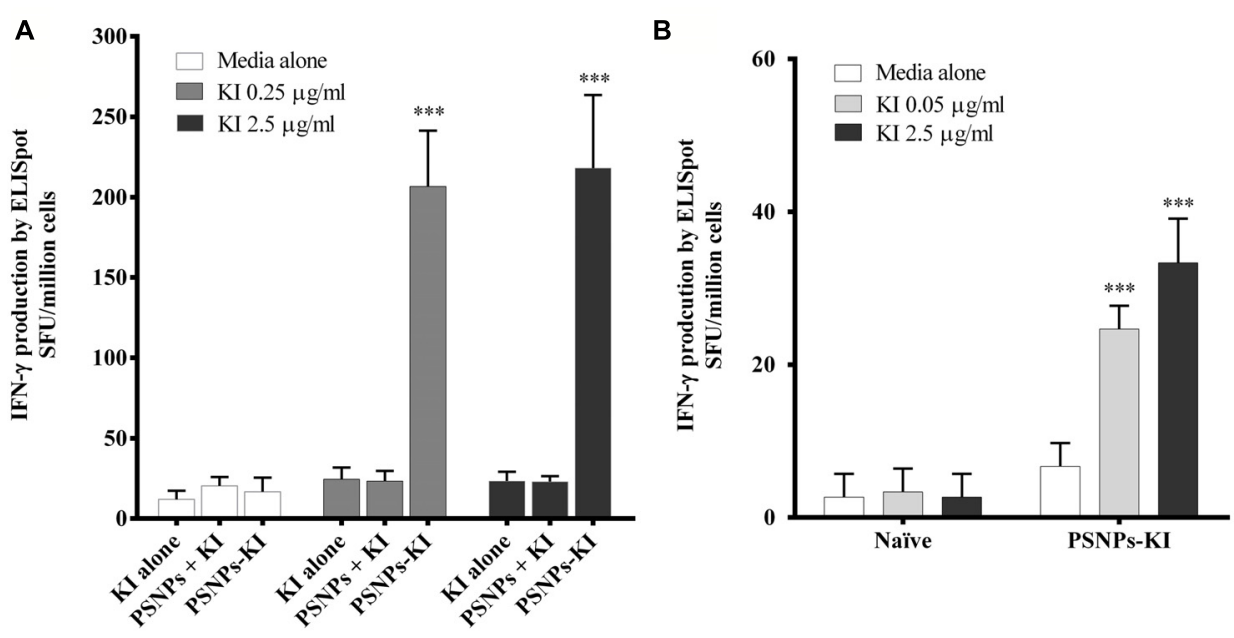

FIGURE 2 | Antigen specific CD8T cell responses induced by SYIPSAEKI peptide vaccines in combination with PSNPs. Mice (BALB/C) were immunized with SYIPSAEKI peptide mixed with or conjugated to PSNPS intradermally at the base of tail. 14 days after the last immunization, spleen cells were collected and assessed for IFN- $\gamma$ production by ELISpot assay. (A) $\mathrm{KI}$ peptides conjugated to, but not mixed with, PSNPs induced high levels of
$\mathrm{KI}$ specific CD8T cell responses after two immunizations (2 weeks apart). Data presented as mean \pm SD of SFU/million cells from each group $(n=3$ mice/group). (B) Immunogenicity of PSNPs-KI formulation after one immunization ( $n=4$ mice per group). Data presented as mean \pm SD of SFU/million cells (pooled for each group) from the triplicated wells in ELISpot assay. Statistical analysis was performed via ANOVA ${ }^{* * *} p \leq 0.001$. in Figure 2B, after one immunization, PSNPs-KI formulations induced good antigen specific CD8 T cell responses, significantly higher than naïve controls $(p<0.001$, Figure 2B). Recall T cells were elicited in ELISpot at both 2.5 and $0.05 \mu \mathrm{g} / \mathrm{ml}$, suggesting high affinity $\mathrm{T}$ cells were induced already in the initial priming phase.

\section{PSNPS-KI AND MONTANIDE INDUCE THE HIGHEST CD8 T CELL RESPONSES}

To benchmark the immunogenicity of PSNPs-KI compared to other types of conventionally adjuvanted experimental formulations capable of inducing CD8 T cell responses, we further tested Montanide and Poly I:C with KI side by side with PSNPs-KI. Strong and comparable KI specific CD8 T cell responses were detected in mice immunized with "PSNPs-KI" and "Montanide + KI" formulations (Figure 3). The magnitude of the KI specific IFN- $\gamma$ production by both these formulations was significantly higher $(p<0.001)$ than that from mice immunized with $\mathrm{KI}$ alone or "Poly I:C + KI" (Figure 3). Despite the literature indicating that Poly I:C is a potent CD8 T cell response inducer (Nordly et al., 2011), Poly I:C mixed with KI formulation didn't promote the induction of IFN- $\gamma$ producing CD8 $\mathrm{T}$ cells above that induced with peptide alone, after two immunizations.

\section{POLY I:C, BUT NOT PSNPS OR MONTANIDE, IS ASSOCIATED WITH A LACK OF DC MATURATION 48 H POST INJECTION WITH THE ADJUVANT ALONE}

To further understand how the potent CD8 T cell responses could be induced by a single CD8 $\mathrm{T}$ cell epitope when conjugated to PSNPs in the absence of a CD4 T cell helper epitope, as well as to compare the non-specific action mode of other adjuvants alone in the induction of cell activation, we investigated the level of DC activation in the local draining lymph node (dLN). This was done by

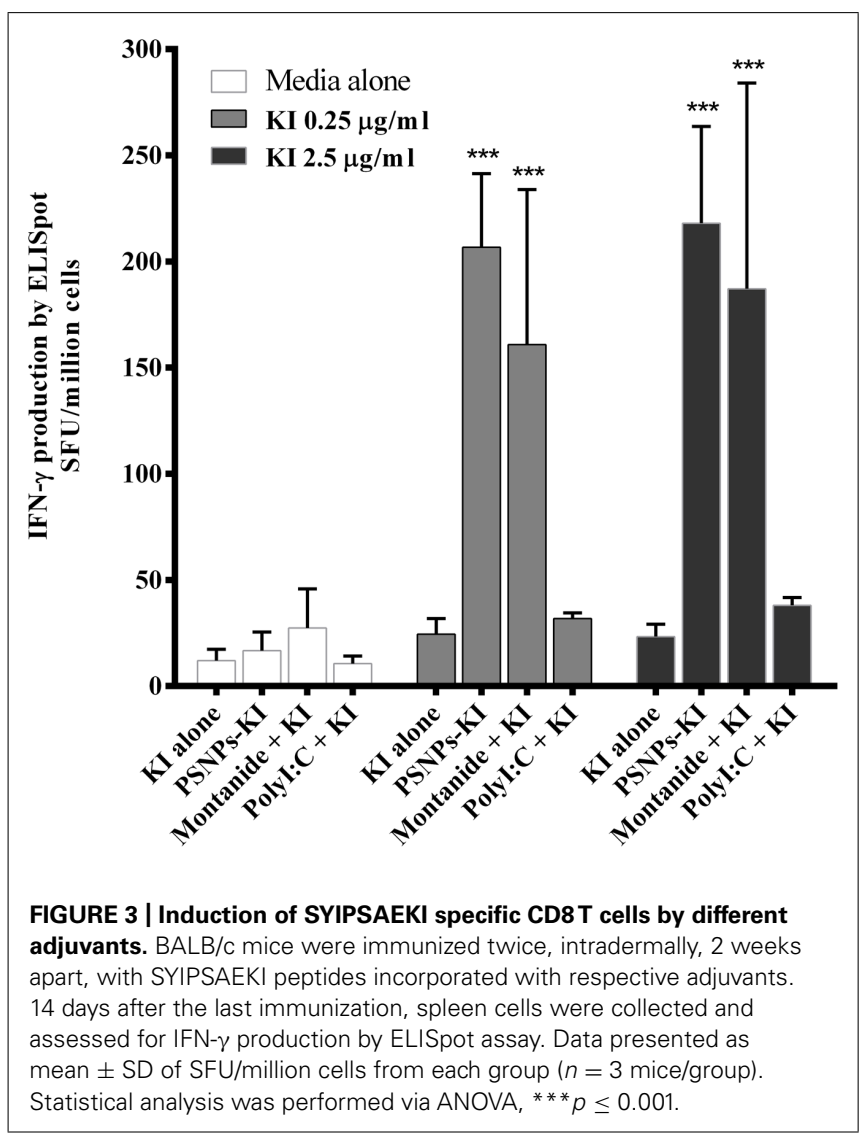

assessing the expression levels of MHCII, CD40, CD80, and CD86 on the CD11c ${ }^{+}$DCs, from the inguinal lymph node, after the injection of PSNPs, Montanide or Poly I:C in vivo, in the absence 
of antigen. We hypothesized that there would be efficient CD86 induction on DC, making them highly capable of activating CD8 T cells (Clarke, 2000; Steinman et al., 2003; Maroof et al., 2009). The critical time-period for CD8 T cell expansion is between 48 and $72 \mathrm{~h}$ post priming, a period of repeated transient contact between T cells and DC (Henrickson et al., 2008). Increases in suppressor cells would be expected to follow initial inflammation induced by adjuvants, which usually peaks at $12-24$ h post administration. Therefore, between 24 and $72 \mathrm{~h}$ immunosuppressive mechanisms would be expected to come into play. We assessed DC frequency and expression of co-stimulatory molecules in adjuvant dLN $48 \mathrm{~h}$ after injection with the adjuvants alone. Results in Figure 4A (gating strategy) and Figure 4B show that the overall frequency of DCs (Gr- ${ }^{-}$CD11c cells) remained the same in the dLN $48 \mathrm{~h}$ post injection with all three types of carrier/adjuvants. There was a significant increase in the expression of CD80 in the dLN DCs after treatment by both PSNPs and Montanide $(p<0.001$ and $p<0.05$, respectively, Figure 4C). Furthermore, there was a significant increase in expression levels of CD86 on CD11 $c^{+}$cells for all adjuvants tested ( $p<0.001$ compared to PSNPs, and $p<0.01$ compared to Montanide and Poly I:C treatment, Figure 4C), implying DCs were potentially being activated even in the absence of a CD4 $\mathrm{T}$ cell helper epitope. CD11c ${ }^{+}$DCs in the Montanide group further showed an increase in the expression of CD40, compared to the naïve and PSNPs groups $(p<0.05$, Figure 4D). However, surprisingly, DCs in the Poly I:C treated group showed significantly lower levels of expression of MHCII compared to all other treatment groups ( $p<0.05$ compared to naïve, $p<0.01$ compared to PSNPs and $p<0.001$ compared to Montanide treatment, Figure 4D), suggesting these DCs were at a different state of maturation, and/or activation, upon treatment with Poly I:C.
A
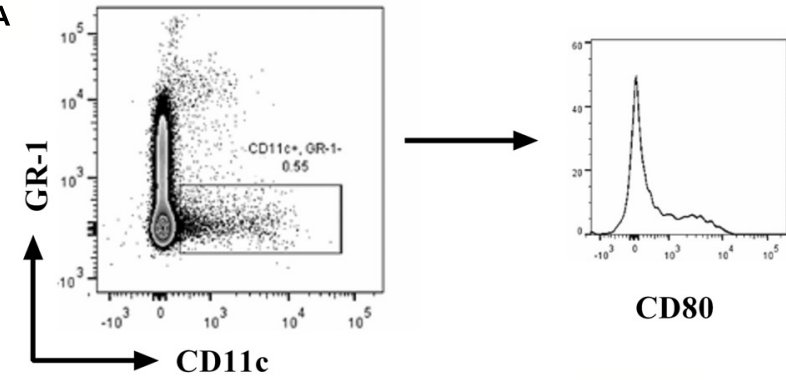

CD80

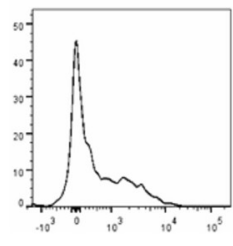

CD86

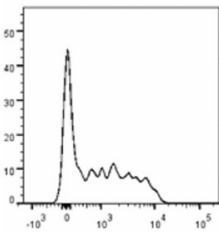

CD40

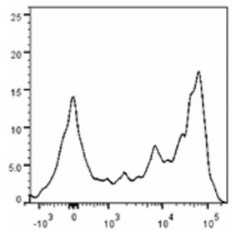

MHCII

C

GR-1 $^{-}$CD11 $c^{+}$

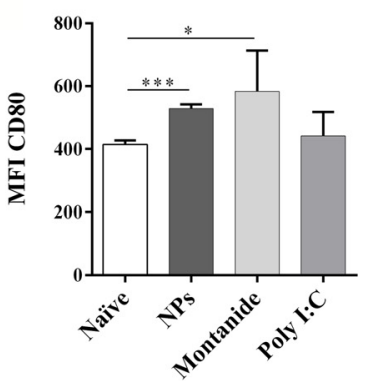

GR-1 ${ }^{-}$CD11 ${ }^{+}$

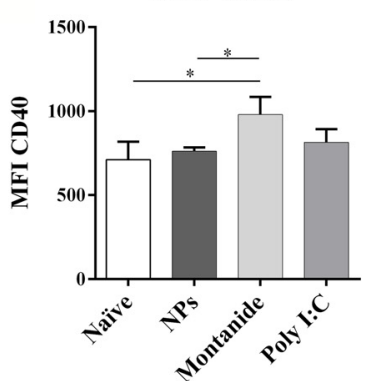

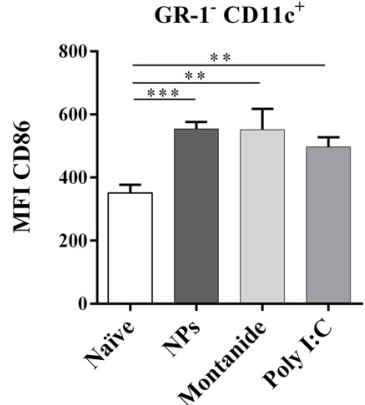

GR-1 ${ }^{-}$CD11 ${ }^{+}$

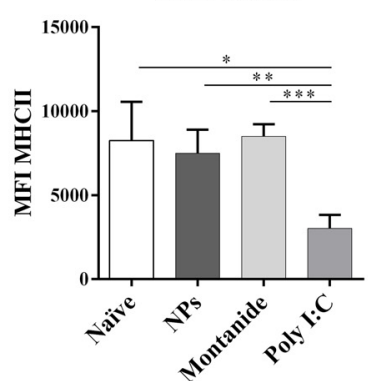

FIGURE 4 | Dendritic cell activation in dLNs after injection with PSNPs, Montanide, and Poly I:C. Mice (BALB/C) were injected once intradermally at the base of tail with the different adjuvants alone. $48 \mathrm{~h}$ after injection, mice were sacrificed, local (inguinal) dLNs were harvested and the levels of $\mathrm{CD}_{11} \mathrm{c}^{+} \mathrm{DC}$ and various activation markers were assessed by flow cytometry. (A) gating strategy; (B) frequency of GR-1-CD11 ${ }^{+}$cells; (C) Mean fluorescent intensity (MFI) of CD80 and CD86 on GR-1- ${ }^{-}$D11 $\mathrm{C}^{+}$ cells; (D) MFI of CD40 and MHCII on GR-1- ${ }^{-}$D $11 \mathrm{c}^{+}$cells. Data presented as mean \pm SD of MFI for each group of treatment ( $n=3$ mice/group). Statistical analysis was performed via $t$-tests, ${ }^{*} p \leq 0.05,{ }^{*} p \leq 0.01,{ }^{*}{ }^{*} p \leq 0.001$. 


\section{PSNPS AND MONTANIDE INDUCE AN ENVIRONMENT THAT} ENCOURAGES STIMULATORY DCs, WHEREAS POLY I:C PROMOTES SUPPRESSIVE MDSCS 48 H POST INJECTION WITH THE ADJUVANTS ALONE

The balance between DCs and MDSCs in the priming lymph node would be predicted to influence the level of immunity subsequently induced by vaccines. We further assessed the MDSC and $\mathrm{CD}_{11 \mathrm{c}^{+}}$DC populations in the dLN $48 \mathrm{~h}$ after injection (Figure 5A). Whilst PSNPs and Montanide maintained a normal MDSC to DC ratio in the dLN (Figure 5B), surprisingly, Poly I:C promoted a significantly higher ratio of MDSCs to DCs, compared to all other groups $(p<0.01$, Figure $5 \mathbf{B})$. Further analysis of the subsets within MDSCs, based on their level of Gr-1 expression, showed that Poly I:C significantly increased the ratio of MDSCs expressing intermediate levels of Gr-1 (monocytic or suppressive MDSC, moMDSC; Kong et al., 2013) over DC, moMDSC/CD11c, when compared to naïve, Montanide or PSNPs treated groups $(p<0.01$, Figure 5C). There was also a significant increase in the ratio of MDSC expressing high levels of Gr-1 (granulocytic MDSC, gMDSC; Kong et al., 2013) to DC (gMDSCs/CD11c) for all treatments $(p<0.01$ compared to naïve and PSNPs, and $p<0.05$ compared to Montanide treatment, Figure 5D), however, the magnitude of this increase was not as high as the increased ratio of
moMDSC/CD11c cells. Hence Poly I:C induced an environment abundant in suppressive (monocytic) phenotype MDSC in the dLN within a short time frame, whereas PSNPs and Montanide did not promote increases in such MDSCs.

\section{POLY I:C AND MONTANIDE PROMOTE THE INDUCTION OF TNFR2+ ${ }^{\text {TREG }}$ CELLS}

Inflammatory, tumor necrosis factor (TNF) inducing, adjuvants such as Montanide and Poly I:C, have the potential to stabilize FoxP3 expression on Treg that express the TNF receptor 2 (TNFR2; Chen and Oppenheim, 2011). TNFR2 has also previously been found to identify the most highly active and immunosuppressive Treg subset (Govindaraj et al., 2014). We speculated that pro-inflammatory adjuvants could therefore increase the Treg to $\mathrm{T}$ effector ratio in the $\mathrm{dLN}$, and if this occurred during the $\mathrm{T}$ cell priming phase, it could potentially interfere with effective CD8 $\mathrm{T}$ cell induction. We further analyzed the Treg and $\mathrm{T}$ effector cells in the dLN (Figure 6A), and found that whilst there was no overall increase in total Treg to $\mathrm{T}$ effector cell ratio $\left(\mathrm{CD} 25^{+}\right.$FoxP3 $^{+}$to $\mathrm{CD}^{2} 5^{-}$FoxP3 $^{-}$cells; Figure 6B), Poly I:C and Montanide significantly increased the frequency of TNFR2 ${ }^{+}$ Treg $\left(\mathrm{FoxP}^{+}{ }^{+} \mathrm{CD} 25^{+} \mathrm{TNFR}^{+}\right.$cells) compared to TNFR2 ${ }^{-}$Treg (FoxP $3^{+} \mathrm{CD}_{25}{ }^{+} \mathrm{TNFR}^{-}$cells) in the $\mathrm{dLN} 48 \mathrm{~h}$ post injection,
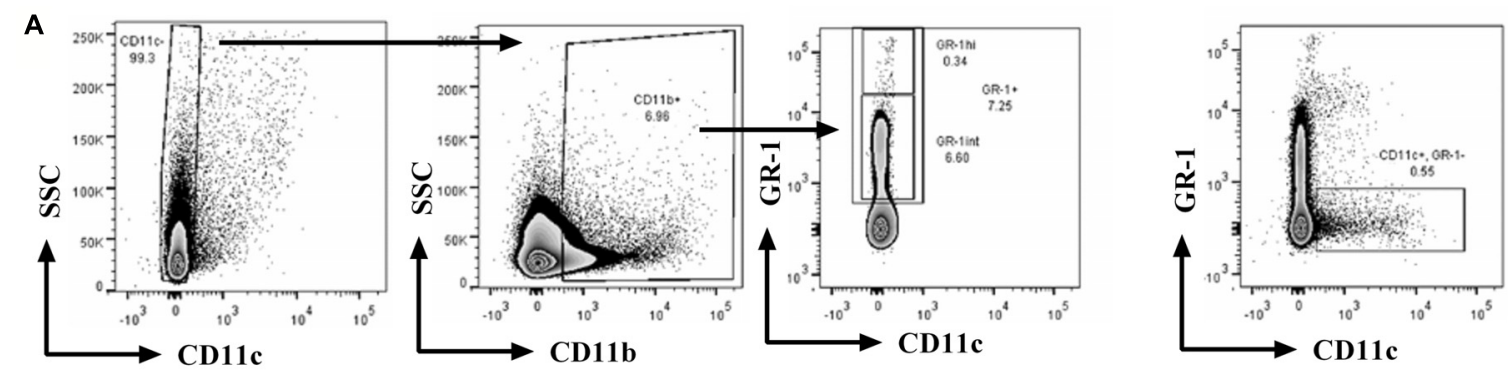

B

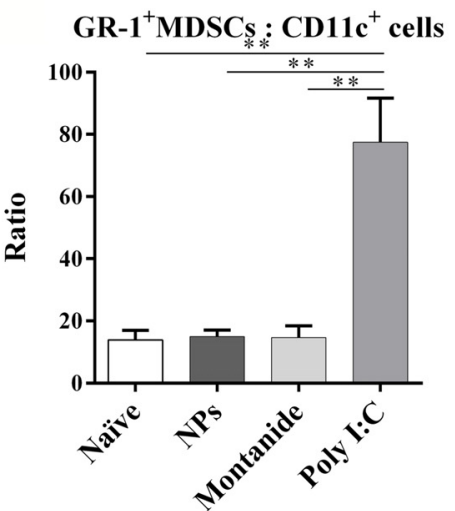

C

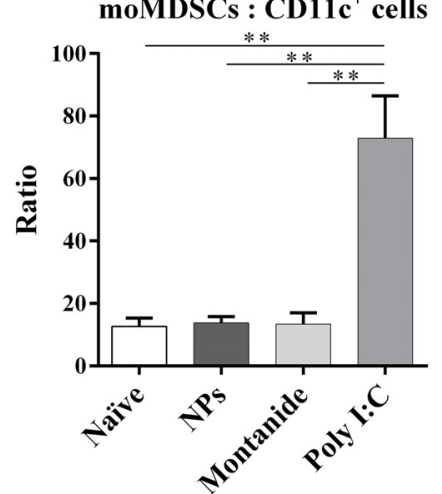

D

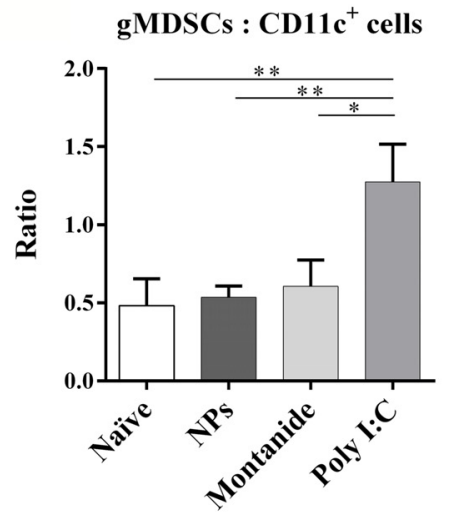

FIGURE 5 | Differential expression of GR-1+ MDSCs and DCs in the local dLN. Mice (BALB/c) were injected once intradermally at the base of the tail with the different adjuvants alone. $48 \mathrm{~h}$ after injection, mice were sacrificed, local (inguinal) dLNs were harvested and levels of GR-1 ${ }^{+}$ MDSCs and DCs, and their ratios, were assessed by flow cytometry.
(A) gating strategy; (B) ratio of GR-1+ MDSCs: $\mathrm{CD}_{11 \mathrm{c}^{+}}$cells; (C) ratio of moMDSCs: CD11c ${ }^{+}$cells; (D) ratio of gMDSCs: CD11c $^{+}$cells. Data presented as mean $\pm S D$ of ratio for each group of treatment $(n=3$ mice/group). Statistical analysis was performed via $t$-tests, ${ }^{*} p \leq 0.05$, ${ }^{*} p \leq 0.01$. 


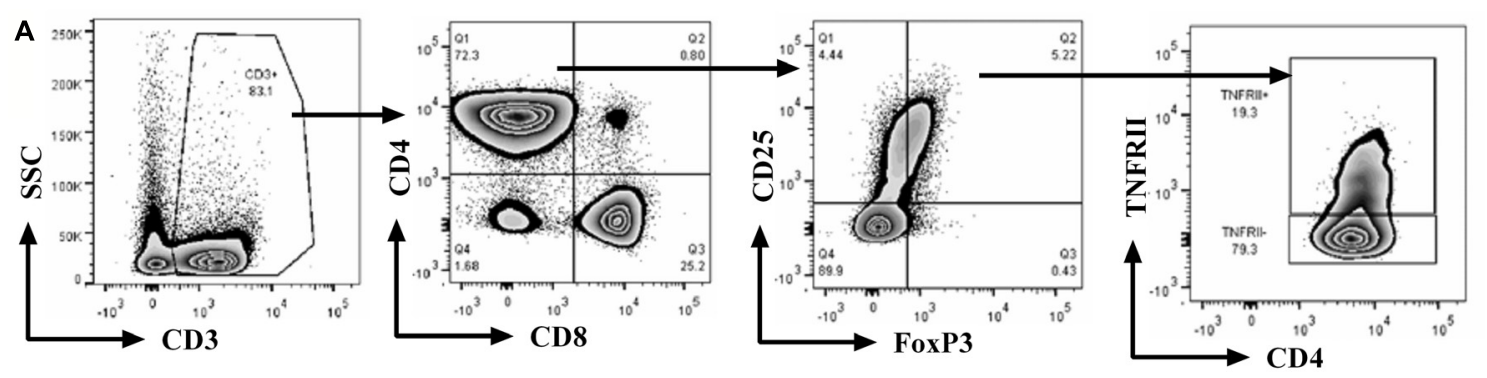

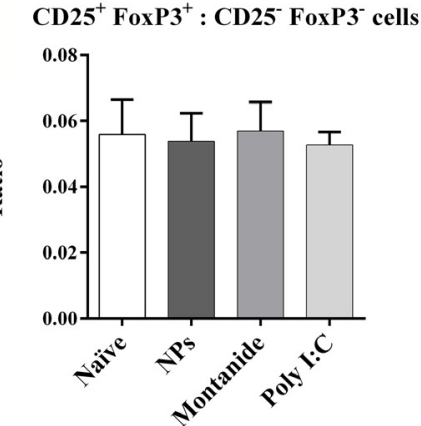

FIGURE 6 | Differential expression of $\mathrm{CD}^{+}{ }^{+}$Treg and effector T cells in the local dLN. Mice (BALB/c) were injected once intradermally at the base of tail with the different adjuvants alone. $48 \mathrm{~h}$ after injection, mice were sacrificed, local (inguinal) dLNs were harvested and the levels of $\mathrm{CD}^{+}+$Treg and effector T cells, and their ratios, were assessed by flow
C $\mathrm{CD25}^{+} \mathrm{FoxP3}^{+} \mathrm{TNFR2}^{+}$: $^{\mathrm{CD}^{+} 5^{+} \mathrm{FoxP3}^{+} \text {TNFR2 }^{-} \text {cells }}$

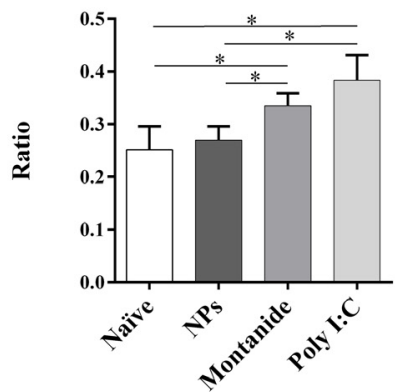

cytometry. (A) gating strategy; (B) ratio of $\mathrm{FoxP}^{+}{ }^{+} \mathrm{CD} 25^{+}$: $\mathrm{FoxP}^{-} \mathrm{CD}^{-} 5^{-}$ cells; (C) ratio of FoxP3 ${ }^{+} \mathrm{CD}_{25} 5^{+} \mathrm{TNFR2} 2^{+}$: FoxP3 ${ }^{+} \mathrm{CD} 25^{+}{ }^{+} \mathrm{TNR} 2^{-}$cells. Data presented as mean \pm SD of ratio for each group of treatment ( $n=3$ mice/group). Statistical analysis was performed via $t$-tests, ${ }^{*} p \leq 0.05$. compared to the naïve and PSNPs groups ( $p<0.05$, Figure 6C). PSNPs maintained the balance of TNFR2 $^{+}$to TNFR2 ${ }^{-}$Treg subpopulations.

\section{DISCUSSION}

The side-by-side comparison of three different adjuvant systems for the induction of highly responsive CD8 T cells to a minimal peptide epitope antigen from CSP of $P$. berghei demonstrated that: (1) Non-inflammatory and inflammatory vaccines can elicit similarly high levels of immune responses, (2) Non-inflammatory nanovaccines require the minimal CD8 T cell epitope peptide to be covalently attached to the nanoparticle carrier, suggesting peptide delivery in vivo is key for antigenic stimulation, (3) Vaccines can prime high levels of CD8 T cells by delivering the minimal CD8 T cell epitope, without helper CD4 epitopes, (4) Inflammatory, but not non-inflammatory, adjuvants result in the induction of TNFR2 ${ }^{+}$Treg in dLNs during a timeframe consistent with the priming of an immune response, and (5) Together with the induction of enhanced numbers of suppressor moMDSC, such findings may explain the particularly poor capacity of Poly I:C to induce CD8 $\mathrm{T}$ cell immune responses.

Both "Montanide + KI" and "PSNPs-KI" formulations induced a similar magnitude of response after two immunizations, reaching the minimum threshold IFN- $\gamma$ production levels determined to be required for sterile protection in the $P$. berghei challenge model (Plebanski et al., 1998). Given a threshold of 100 spots was required to start seeing sterile protection in about $74 \%$ of animals in previous studies (Plebanski et al., 1998), it is likely that the 200 spots achieved by the nanovaccines would also be protective, although it will be important to confirm this formally. The potentially protective IFN- $\gamma$ levels produced in this study merit additional validation in further direct challenge studies. There may be additional advantages in using a non-inflammatory nanoparticle approach over Montanide. Montanide is a viscous combination of adjuvant with peptide, creating a depot at the injection site with the antigen, associated with some pain and local inflammation. As well as increasing compliance with vaccination, the use of a non-inflammatory adjuvant system that substantially drains to the lymph nodes, may, in the case of immunization of individuals in malaria endemic areas, help minimize the risk of triggering inflammatory feedback loops, such as those associated with cerebral malaria. Previous studies have shown nanoparticle based vaccines do not need to engage conventional inflammatory pathways to induce adaptive immunity (Karlson Tde et al., 2013; Xiang et al., 2013), and act by selectively targeting DCs, particularly $\mathrm{CD}^{+}$DCs, directly in the local lymph nodes (Fifis et al., 2004a; Mottram et al., 2007; Xiang et al., 2013), as well as by promoting uptake by DC in the periphery followed by subsequent migration via the afferent lymphatics (Gamvrellis et al., 2013). The critical factor identified that promotes $\mathrm{CD}^{+}$DCs targeting was found to be particle size (40-50 nm; Fifis et al., 2004a; Mottram et al., 2007). The fact that mixed-in nanoparticles in this study did not 
act as conventional adjuvants, and hence the carrier activity of the nanoparticles was sufficient and necessary to induce high levels of immune responses, predicts that nanoparticle carriers of the correct size to target $\mathrm{CD}^{+}$DCs in vivo (made of non-inflammatory materials) would also be capable of inducing high levels of immunity. Given the explosion in nanomaterials and delivery systems, this appears to be a promising and timely finding.

It was surprising to find that the "Poly I:C + KI" formulation was unable to induce similarly high CD8 $\mathrm{T}$ cell responses when compared side-by-side with the "Montanide + KI" and "PSNPsKI" formulations. This could be mechanistically explained by the new finding that Poly I:C promotes dramatic increases in the ratio of MDSCs to DCs, including moMDSCs, in the LNs draining the injection site, within a timeframe capable of interfering with local CD8 T cell priming. Moreover, whereas the frequency of DCs remained the same in the dLN $48 \mathrm{~h}$ post injection with either PSNPs, Montanide or Poly I:C, there were significantly lower levels of MHCII expression on DCs treated with Poly I:C. Down-regulation of some activation markers on DCs has been associated in the literature with increases in suppressor MDSC frequencies and their subsequent apoptosis (Shen et al., 2014). MDSCs can suppress effector T cell responses directly, or by promoting the expansion of Tregs in the presence of IFN- $\gamma$ (Kong et al., 2013).

Together our results suggest that non-inflammatory nanoparticles 40-50 nm or Montanide can be used to induce potent CD8 $\mathrm{T}$ cell responses, even when used with purely a minimal CD8 $\mathrm{T}$ cell peptide epitope. Generally, the results herein also suggest a new paradigm for highly immunogenic vaccines, which could instead of delivering pro-inflammatory danger signals, be designed to 'keep under the radar' to deliver antigen to crosspriming $\mathrm{CD}^{+}{ }^{+}$DCs whilst avoiding the expansion of some key immunosuppressive and inflammation reactive cell populations.

\section{ACKNOWLEDGMENTS}

The authors wish to thank Ms. Ying Ying Kong and Mr. Mutsa Madondo for their technical support on flow cytometry analysis and the staff at MICU animal facility for taking care of all mice.

\section{REFERENCES}

Arama, C., and Troye-Blomberg, M. (2014). The path of malaria vaccine development: challenges and perspectives. J. Intern. Med. 275, 456-466. doi: 10.1111/joim.12223

Aucouturier, J., Dupuis, L., Deville, S., Ascarateil, S., and Ganne, V. (2002). Montanide ISA 720 and 51: a new generation of water in oil emulsions as adjuvants for human vaccines. Expert Rev. Vaccines 1, 111-118. doi: 10.1586/14760584.1. 1.111

Bonhoure, F., and Gaucheron, J. (2006). Montanide ISA 51 VG as adjuvant for human vaccines. J. Immunother. 29, 647-648.

Chen, X., and Oppenheim, J. J. (2011). Contrasting effects of TNF and anti-TNF on the activation of effector $\mathrm{T}$ cells and regulatory $\mathrm{T}$ cells in autoimmunity. FEBS Lett. 585, 3611-3618. doi: 10.1016/j.febslet.2011.04.025

Clarke, S. R. (2000). The critical role of CD40/CD40L in the CD4-dependent generation of $\mathrm{CD}^{8+} \mathrm{T}$ cell immunity. J. Leukoc. Biol. 67, 607-614.

Doolan, D. L., and Martinez-Alier, N. (2006). Immune response to preerythrocytic stages of malaria parasites. Curr. Mol. Med. 6, 169-185. doi: $10.2174 / 156652406776055249$

Fifis, T., Gamvrellis, A., Crimeen-Irwin, B., Pietersz, G. A., Li, J., Mottram, P. L., et al. (2004a). Size-dependent immunogenicity: therapeutic and protective properties of nano-vaccines against tumors. J. Immunol. 173, 3148-3154. doi: 10.4049/jimmunol.173.5.3148
Fifis, T., Mottram, P., Bogdanoska, V., Hanley, J., and Plebanski, M. (2004b) Short peptide sequences containing MHC class I and/or class II epitopes linked to nano-beads induce strong immunity and inhibition of growth of antigen-specific tumour challenge in mice. Vaccine 23, 258-266. doi: 10.1016/j.vaccine.2004.05.022

Gamvrellis, A., Walsh, K., Tatarczuch, L., Smooker, P., Plebanski, M., and Scheerlinck, J. P. (2013). Phenotypic analysis of ovine antigen presenting cells loaded with nanoparticles migrating from the site of vaccination. Methods 60, 257-263. doi: 10.1016/j.ymeth.2013.02.008

Govindaraj, C., Tan, P., Walker, P., Wei, A., Spencer, A., and Plebanski, M. (2014). Reducing TNF receptor $2+$ regulatory $\mathrm{T}$ cells via the combined action of azacitidine and the HDAC inhibitor, panobinostat for clinical benefit in acute myeloid leukemia patients. Clin. Cancer Res. 20, 724-735. doi: 10.1158/1078-0432.CCR-13-1576

Henrickson, S. E., Mempel, T. R., Mazo, I. B., Liu, B., Artyomov, M. N., Zheng, H., et al. (2008). In vivo imaging of $\mathrm{T}$ cell priming. Sci. Signal. 1, pt2. doi: $10.1126 /$ stke.112pt2

Karlson Tde, L., Kong, Y. Y., Hardy, C. L., Xiang, S. D., and Plebanski, M. (2013). The signalling imprints of nanoparticle uptake by bone marrow derived dendritic cells. Methods 60, 275-283. doi: 10.1016/j.ymeth.2013.02.009

Kong, Y. Y., Fuchsberger, M., Xiang, S. D., Apostolopoulos, V., and Plebanski, M. (2013). Myeloid derived suppressor cells and their role in diseases. Curr. Med. Chem. 20, 1437-1444. doi: 10.2174/0929867311320110006

Krzych, U., Zarling, S., and Pichugin, A. (2014). Memory T cells maintain protracted protection against malaria. Immunol. Lett. 161, 189-195. doi: 10.1016/j.imlet.2014.03.011

Longhi, M. P., Trumpfheller, C., Idoyaga, J., Caskey, M., Matos, I., Kluger, C., et al. (2009). Dendritic cells require a systemic type I interferon response to mature and induce $\mathrm{CD}^{4+}$ Th1 immunity with poly IC as adjuvant. J. Exp. Med. 206, 1589-1602. doi: 10.1084/jem.20090247

Maroof, A., Beattie, L., Kirby, A., Coles, M., and Kaye, P. M. (2009). Dendritic cells matured by inflammation induce CD86-dependent priming of naive $\mathrm{CD}^{8+} \mathrm{T}$ cells in the absence of their cognate peptide antigen. J. Immunol. 183, 7095-7103. doi: 10.4049/jimmunol.0901330

Mbow, M. L., De Gregorio, E., Valiante, N. M., and Rappuoli, R. (2010). New adjuvants for human vaccines. Curr. Opin. Immunol. 22, 411-416. doi: 10.1016/j.coi.2010.04.004

Mottram, P. L., Leong, D., Crimeen-Irwin, B., Gloster, S., Xiang, S. D., Meanger, J., et al. (2007). Type 1 and 2 immunity following vaccination is influenced by nanoparticle size: formulation of a model vaccine for respiratory syncytial virus. Mol. Pharm. 4, 73-84. doi: 10.1021/mp060096p

Nordly, P., Rose, F., Christensen, D., Nielsen, H. M., Andersen, P., Agger, E. M., et al. (2011). Immunity by formulation design: induction of high $\mathrm{CD}^{8+} \mathrm{T}$ cell responses by poly(I:C) incorporated into the CAF01 adjuvant via a double emulsion method. J. Control. Release 150, 307-317. doi: 10.1016/j.jconrel.2010. 11.021

Ohkusu-Tsukada, K., Ohta, S., Kawakami, Y., and Toda, M. (2011). Adjuvant effects of formalin-inactivated HSV through activation of dendritic cells and inactivation of myeloid-derived suppressor cells in cancer immunotherapy. Int. J. Cancer 128, 119-131. doi: 10.1002/ijc.25319

Perret, R., Sierro, S. R., Botelho, N. K., Corgnac, S., Donda, A., and Romero, P. (2013). Adjuvants that improve the ratio of antigen-specific effector to regulatory T cells enhance tumor immunity. Cancer Res. 73, 6597-6608. doi: 10.1158/00085472.CAN-13-0875

Plebanski, M., Gilbert, S. C., Schneider, J., Hannan, C. M., Layton, G., Blanchard, T., et al. (1998). Protection from Plasmodium berghei infection by priming and boosting $\mathrm{T}$ cells to a single class I-restricted epitope with recombinant carriers suitable for human use. Eur. J. Immunol. 28, 4345-4355. doi: 10.1002/(SICI)15214141(199812)28:12\&\#60;4345::AID-IMMU4345\&\#62;3.0.CO;2-P

Plebanski, M., Lopez, E., Proudfoot, O., Cooke, B. M., Itzstein, M., and Coppel, R. L. (2006). Economic and practical challenges to the formulation of vaccines against endemic infectious diseases such as malaria. Methods 40, 77-85. doi: 10.1016/j.ymeth.2006.05.021

Postels, D. G., and Birbeck, G. L. (2013). Cerebral malaria. Handb. Clin. Neurol. 114, 91-102. doi: 10.1016/B978-0-444-53490-3.00006-6

Schneider, J., Gilbert, S. C., Hannan, C. M., Degano, P., Prieur, E., Sheu, E. G., et al. (1999). Induction of $\mathrm{CD}^{8+} \mathrm{T}$ cells using heterologous prime-boost immunisation strategies. Immunol. Rev. 170, 29-38. doi: 10.1111/j.1600-065X.1999.tb0 1326.x 
Shen, J., Chen, X., Wang, Z., Zhang, G., and Chen, W. (2014). Downregulation of CD40 expression contributes to the accumulation of myeloid-derived suppressor cells in gastric tumors. Oncol. Lett. 8, 775-780. doi: 10.3892/ol.2014.2174

Steinman, R. M., Hawiger, D., Liu, K., Bonifaz, L., Bonnyay, D., Mahnke, K., et al. (2003). Dendritic cell function in vivo during the steady state: a role in peripheral tolerance. Ann. N. Y. Acad. Sci. 987, 15-25. doi: 10.1111/j.17496632.2003.tb06029.x

Trumpfheller, C., Caskey, M., Nchinda, G., Longhi, M. P., Mizenina, O., Huang, Y., et al. (2008). The microbial mimic poly IC induces durable and protective $\mathrm{CD}^{4+}$ T cell immunity together with a dendritic cell targeted vaccine. Proc. Natl. Acad. Sci. U.S.A. 105, 2574-2579. doi: 10.1073/pnas.0711976105

Xiang, S. D., Kalkanidis, M., Pietersz, G. A., Mottram, P. L., Crimeen-Irwin, B., Ardipradja, K., et al. (2006). Methods for nano-particle based vaccine formulation and evaluation of their immunogenicity. Methods 40, 20-29. doi: 10.1016/j.ymeth.2006.05.018

Xiang, S. D., Wilson, K., Day, S., Fuchsberger, M., and Plebanski, M. (2013). Methods of effective conjugation of antigens to nanoparticles as non-inflammatory vaccine carriers. Methods 60, 232-241. doi: 10.1016/j.ymeth.2013.03.036
Conflict of Interest Statement: The authors declare that the research was conducted in the absence of any commercial or financial relationships that could be construed as a potential conflict of interest.

Received: 22 October 2014; paper pending published: 09 November 2014; accepted: 09 January 2015; published online: 06 February 2015.

Citation: Wilson KL, Xiang SD and Plebanski M (2015) Montanide, Poly I:C and nanoparticle based vaccines promote differential suppressor and effector cell expansion: a study of induction of CD8 T cells to a minimal Plasmodium berghei epitope. Front. Microbiol. 6:29. doi: 10.3389/fmicb.2015.00029

This article was submitted to Microbial Immunology, a section of the journal Frontiers in Microbiology.

Copyright (C) 2015 Wilson, Xiang and Plebanski. This is an open-access article distributed under the terms of the Creative Commons Attribution License (CC BY). The use, distribution or reproduction in other forums is permitted, provided the original author(s) or licensor are credited and that the original publication in this journal is cited, in accordance with accepted academic practice. No use, distribution or reproduction is permitted which does not comply with these terms. 